\title{
Clinicoradiological features and surgical outcomes of primary intracranial medulloepitheliomas: a single-center experience and pooled analysis of individual patient data
}

\author{
Da Li, MD, ${ }^{1}$ Shu-Yu Hao, MD, PhD, ${ }^{1}$ Liang Wang, MD, PhD, ${ }^{1}$ Gui-Lin Li, MD, PhD, ${ }^{2}$ \\ Jun-Mei Wang, MD, PhD, ${ }^{2}$ Zhen Wu, MD, PhD, ${ }^{1}$ Li-Wei Zhang, MD, PhD, ${ }^{1}$ Jun-Ting Zhang, MD, ${ }^{1}$ and \\ Wang Jia, MD, PhD'
}

'Department of Neurosurgery, Beijing Tiantan Hospital, Capital Medical University; and 'Department of Neuropathology, Beijing Neurosurgical Institute, Beijing, People's Republic of China

\begin{abstract}
OBJECTIVE Medulloepithelioma (MEPL) is a rare, malignant primitive neuroectodermal tumor with dismal survival rates. The authors aimed to define independent risk factors for progression-free survival (PFS) and overall survival (OS) and to propose an optimal treatment protocol for MEPL.

METHODS The authors reviewed the clinicoradiological data obtained in 12 patients with MEPL who underwent surgical treatment at their institution between January 2008 and June 2016. In addition, they reviewed 55 cases of MEPL published in the literature from January 1957 to July 2017. A pooled analysis of individual patient data of these 67 patients was performed to evaluate risk factors.
\end{abstract}

RESULTS The authors' cohort included 5 males and 7 females with a mean age of 15.7 years. Gross-total resection (GTR) was achieved in 10 (83.3\%) patients. Radiotherapy (mean total dose $42.8 \mathrm{~Gy}$ ) and chemotherapy were administered to 7 and 4 patients, respectively. After a median follow-up of 21.7 months, $6(50 \%)$ patients suffered recurrence and subsequently died, with median PFS and OS times of 5.5 and 13.9 months, respectively. Among the 55 patients in the literature, 13 (23.6\%) patients received GTR, and $25(49.0 \%)$ and 15 (29.4\%) received radiotherapy (median total dose $53.2 \mathrm{~Gy}$ ) and chemotherapy, respectively. After a median follow-up of 10.0 months, the recurrence and mortality rates were $69.7 \%$ (23/33) and 70.8\% (34/48), respectively, and the median PFS was 6.0 months. Of the pooled cohort, the actuarial 5 -year PFS and OS were $36.3 \%$ and $29.2 \%$, respectively, and the estimated median survival time for PFS and OS were 12.8 and 15.2 months, respectively. A multivariate Cox model verified non-GTR (HR 5.537, $p<0.001$ ) and no radiotherapy (HR 3.553, $p=0.008$ ) as independent adverse factors for PFS. The 5-year PFS in patients with or without GTR was $63.8 \%$ and $6.3 \%$, respectively, and in patients with or without radiotherapy was $42.7 \%$ and $23.1 \%$, respectively. A multivariate model demonstrated non-GTR (HR 9.089, $p<0.001$ ), no radiotherapy (HR 3.126, p = 0.004), and no chemotherapy (HR 3.621, $p=0.004$ ) as independent adverse factors for poor OS. The 5-year OS in patients with GTR, radiotherapy, or chemotherapy was $72.1 \%, 44.0 \%$, and $58.0 \%$, respectively. In contrast, in patients without GTR, radiotherapy, or chemotherapy, the 5 -year OS was $5.8 \%, 14.3 \%$, and $15.8 \%$, respectively. Overall, in patients receiving GTR plus chemoradiotherapy, the actuarial 5-year PFS and OS were both $87.5 \%$.

CONCLUSIONS MEPL is a rare neoplastic entity with a poor prognosis. There are no distinguishing radiological features apart from cystic degeneration. Via the pooled analysis, the authors identified independent adjustable factors associated with PFS and OS, from which they advocate for GTR plus chemoradiotherapy with a sufficient dose if tolerable as an optimal treatment to improve outcomes. Future studies with large cohorts will be necessary to verify our findings.

https://thejns.org/doi/abs/10.3171/2018.1.JNS172509

KEYWORDS chemotherapy; medulloepithelioma; primitive neuroectodermal tumor; radiotherapy; total resection; oncology

ABBREVIATIONS AUPBSCT = autologous peripheral blood stem cell transplantation; CSI = craniospinal irradiation; GTR = gross-total resection; KPS = Karnofsky Performance Scale; MEPL = medulloephithelioma; OS = overall survival; PFS = progression-free survival; $P R=$ partial resection; STR = subtotal resection.

SUBMITTED October 4, 2017. ACCEPTED January 19, 2018.

INCLUDE WHEN CITING Published online July 6, 2018; DOI: 10.3171/2018.1.JNS172509. 
$\mathrm{M}$ EDULLOEPITHELIOMA (MEPL) is a rare, primitive neuroectodermal tumor with extremely malignant biological behavior and dismal survival rates. It displays features of the embryonal neural tube formation and often occurs in childhood and occasionally in adults. ${ }^{15,29}$ MEPL was first reported by Bailey and Cushing in $1926^{2}$ and was reported to have the potential capacity for divergent differentiation along neuronal, astrocytic, and ependymal lines. ${ }^{29}$ Due to the rarity of MEPL, the clinicoradiological characteristics and optimal treatment protocols remain undefined, and risk factors for poor longterm outcomes are not well described. Clinical descriptions of MEPL can be gleaned only from small series and case reports. A recent study by Müller et al. ${ }^{24}$ identified age younger than 4 years and non-gross total resection (GTR) as adverse factors for overall survival (OS). However, no specific treatment modality was recommended, and the usefulness of radiotherapy or chemotherapy remains unclear. Successful treatment of MEPL with favorable prognosis in a single case report is less convincing and has limited potential for extrapolation to larger numbers of patients with MEPL. Therefore, the present study aimed to detail the clinicoradiological features of MEPL, evaluate the risk factors for progression-free survival (PFS) and OS, and propose a treatment protocol based on our experience and the existing literature.

\section{Methods}

\section{Clinical and Radiographic Data}

This study included 12 patients with intracranial MEPLs who were surgically treated and pathologically confirmed at Beijing Tiantan Hospital between January 2008 and June 2016. The Beijing Tiantan Hospital research ethics committee approved this study. The clinical charts, radiographic data, and surgical records were retrospectively reviewed. Pre- and postoperative neurological statuses were evaluated using the Karnofsky Performance Scale (KPS). The following information was recorded: age, sex, preoperative symptoms, symptom duration, preoperative diagnosis, tumor characteristics (including size, location, boundary, and enhancement), surgical findings, extent of tumor removal, and postoperative radiotherapy and/or chemotherapy. Tumor size was evaluated and expressed as the lesion equivalent diameter $\left[(\mathrm{abc})^{1 / 3}\right]$, where $\mathrm{a}, \mathrm{b}$, and $\mathrm{c}$ represent the diameters measured on the axial, sagittal, and coronal MRI scans (Fig. 1), respectively. The extent of tumor removal was recorded as GTR, subtotal resection (STR), or partial resection (PR) based on postoperative contrast MRI (Tables 1 and 2). GTR was defined as complete removal without residual.

\section{Pooled Analysis of Individual Patient Data}

For the pooled analysis of individual patient data regarding intracranial MEPL, a search was conducted of the English-language literature related to MEPL in the PubMed database from January 1957 to July 2017. The keyword used in the search was "medulloepithelioma." We further reviewed all of the references provided in the identified publications and incorporated all of the pertinent citations. Cases that were included contained ad-
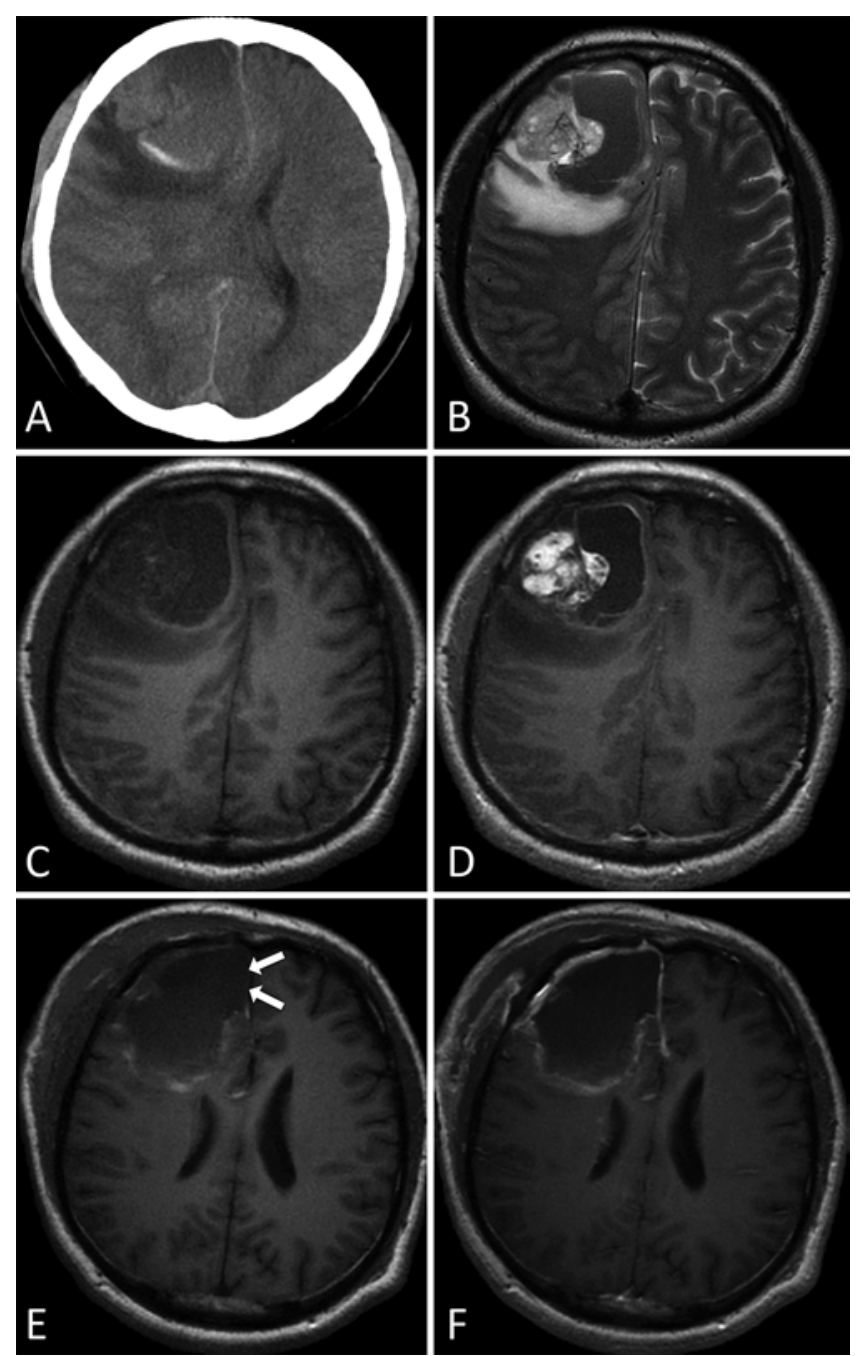

FIG. 1. Case 2. A: Preoperative CT scan revealing a right frontal lobe lesion with intralesional hemorrhage, peritumoral edema, and a mass effect. B-D: Preoperative axial T2-weighted (B), T1-weighted (C), and contrast-enhanced (D) MRI scans showing a cystic-solid lesion with enhancement. $\mathbf{E}$ and F: Postoperative axial T1-weighted $(\mathrm{E})$ and contrast-enhanced (F) MRI scans showing total resection. In this case, total resection was evaluated according to the following signs. 1) The main solid and cystic components had been completely removed. 2) The resected extent had been extended and included regions approximately $0.5-1.0 \mathrm{~cm}$ surrounding the contrasted lesions and the cyst wall. 3) The peritumoral edema zone had been removed, which was absent on the postoperative MRI scans. 4) The frontopolar cortex and the anteromedial cyst wall (arrows in E) adjacent to the falx had been totally removed. 5) The notable contrast enhancement along the surgical cavity wall was significantly more intense and thicker than that in the preoperative cyst wall, and the postoperative radiological features after contrast enhancement were more likely associated with postoperative changes (i.e., minor bleeding and hemostasis material) rather than residuals; additionally, on the postoperative T1-weighted image (E), the hyperintense signal along the surgical cavity wall suggested postoperative changes that were quite different from signals of the preoperative MRI. 6) Because of an absence of parenchyma, the contrast enhancement along the falx and the frontopolar dura ( $F$ ) precluded the possibility of residual. 7) Finally, most importantly, resection of this lesion was performed along the boundary between tumor and normal parenchyma, followed by extended resection that guaranteed total resection. 
Li et al.

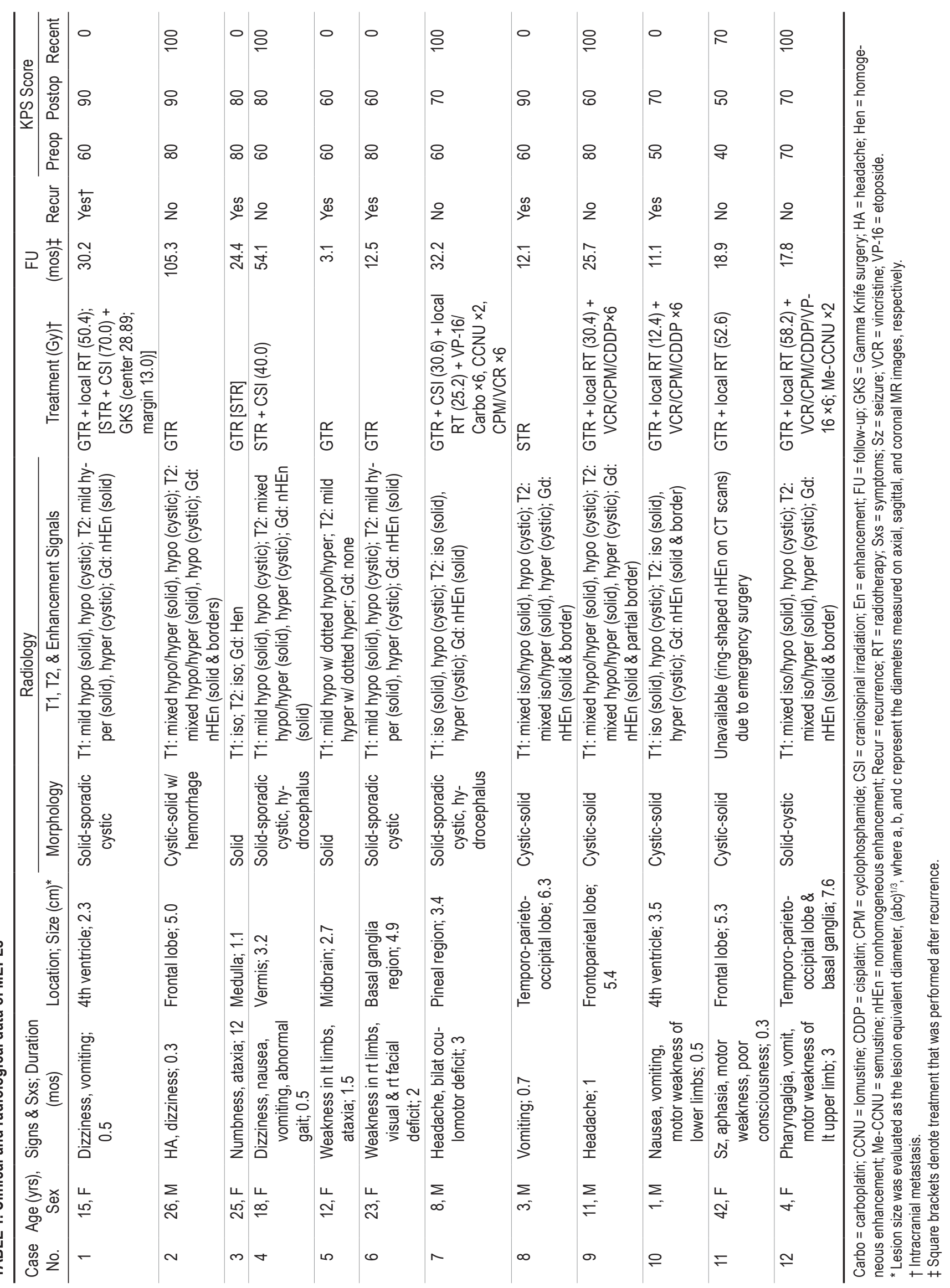


equate clinical data pertinent to the analysis and were reported in the English language (Table 3).

\section{Pathological Examination}

Fresh paraffin-embedded tumor tissue was cut into 5- $\mu \mathrm{m}$ slices and stained with H \& E (Fig. 2). Immunohistochemical staining for CD30, CD56, CD99, cytokeratin, epithelial membrane antigen (EMA), GFAP, Ki-67/MIB1, MAP-2, nestin, neurofilament, NeuN, neuron-specific enolase (NSE), Olig2, S100, synaptophysin (SYN), and vimentin was used for differential diagnosis of the samples. Two independent neuropathologists (J.M.W. and G.L.L.) reviewed the microscopic pathologies of the MEPL samples. Diagnosis was established based on the typical histopathological characteristics of MEPL, including embryonal neural tube-like formation delimited by pseudostratified columnar epithelia and occasional differentiation into neuronal, glial, and mesenchymal elements.

\section{Follow-Up and Statistical Analysis}

Due to the pathological malignancy, radiotherapy and chemotherapy were recommended to all patients based on consultation with oncologists and radiologists. The choice of dosing of the radiotherapy was based on the principles of radiation therapy for high-risk medulloblastoma, and the dose was 36 Gy craniospinal irradiation (CSI) in 20 fractions with boosting to the primary brain site to 54-55.8 Gy in 27-31 fractions. During CSI, weekly administration of vincristine was recommended, followed by either of the following protocols: 1) cisplatin, cyclophosphamide, and vincristine; and 2) cisplatin, lomustine, and vincristine. Scheduled radiographic follow-up with contrast-enhanced MRI at 3, 6, and 12 months after surgery and then once every 6 months thereafter was advised for monitoring the effectiveness of radiotherapy and/or chemotherapy. Any treatments for recurrence were noted, as was the cause of death if the patient died.

With the combined prior cases $(n=55)$ and present series $(n=12)($ Table 4$)$, the primary outcomes of MEPL were PFS, OS, and associated adverse factors evaluated by both uni- and multivariate Cox regression models, and risk factors are detailed in Tables 5 and 6 . The survival curves of patients with significant risk factors were illustrated using the Kaplan-Meier method. Because the percentage of censored cases in some subgroups exceeded $50 \%$, the estimated median survival time was not provided; therefore, the estimated mean survival times are cited in our study. Chi-square values in our Kaplan-Meier curves were obtained by log-rank (Mantel-Cox) testing during the Kaplan-Meier curve analysis. The cutoff value for an age of 6 years was determined by receiver operator characteristic (ROC) curve analysis. Analyses were performed using IBM SPSS statistical package software (version 20.0, IBM Corp.) with the significance set at $\mathrm{p}<0.05$.

\section{Results}

\section{Patient Demographics}

The present series included 5 males and 7 females with a mean age of 15.7 years (range 1-42 years). The mean duration of symptoms was 2.1 months (range 0.3-12.0

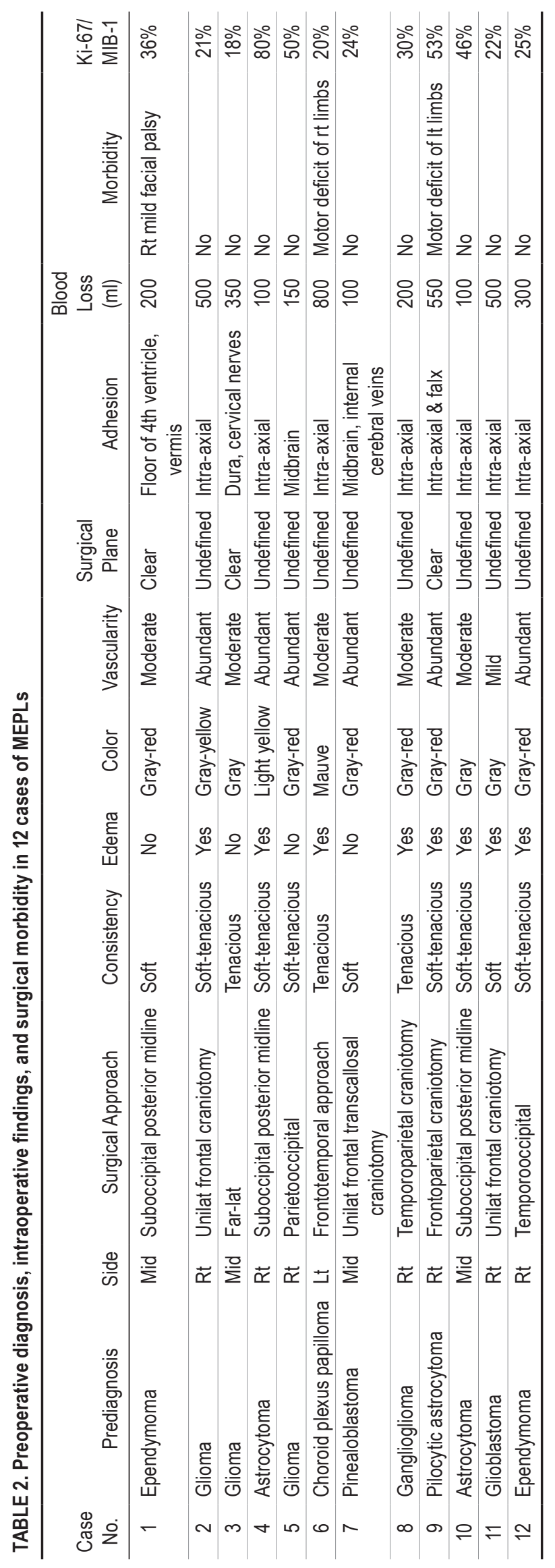


Li et al.

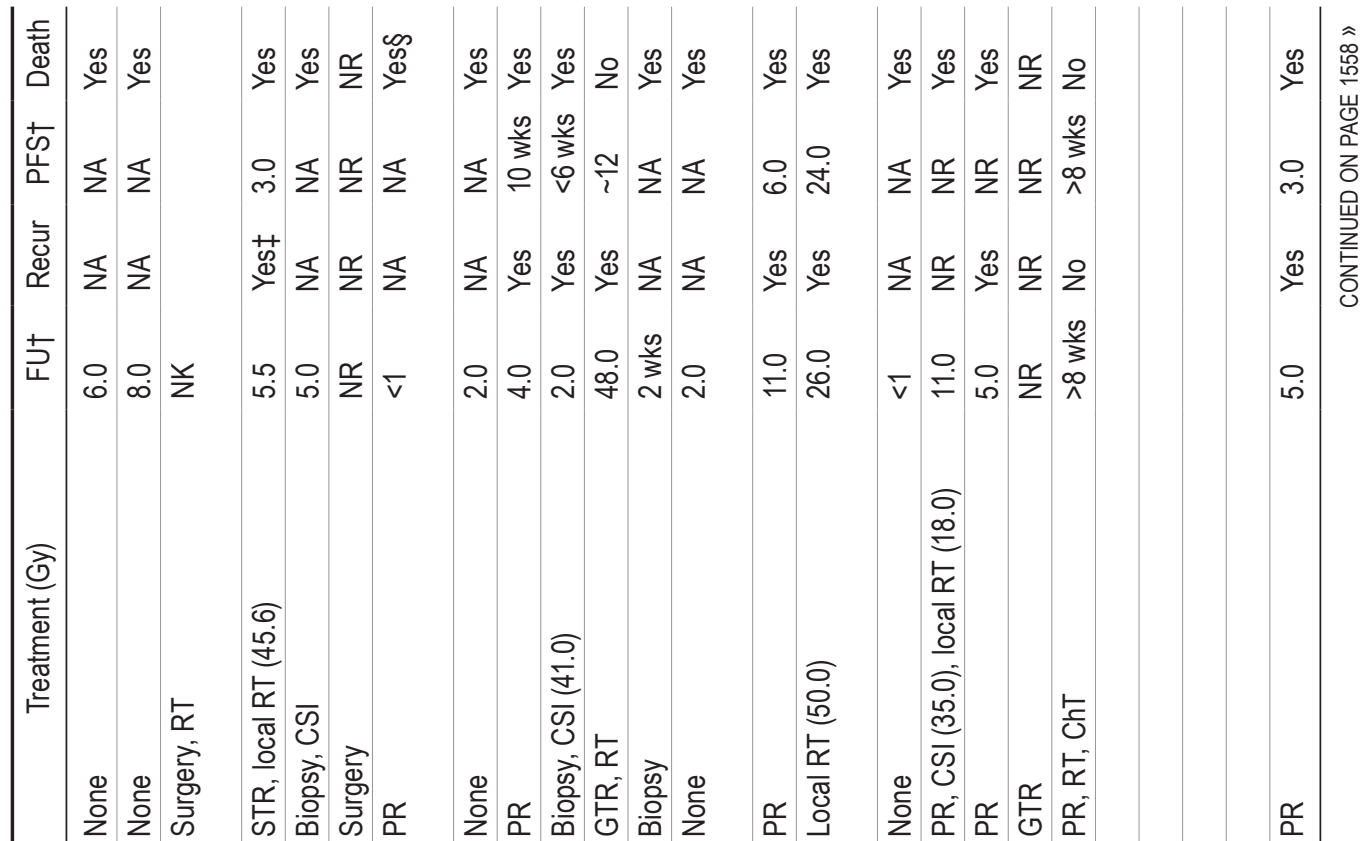

离

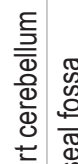

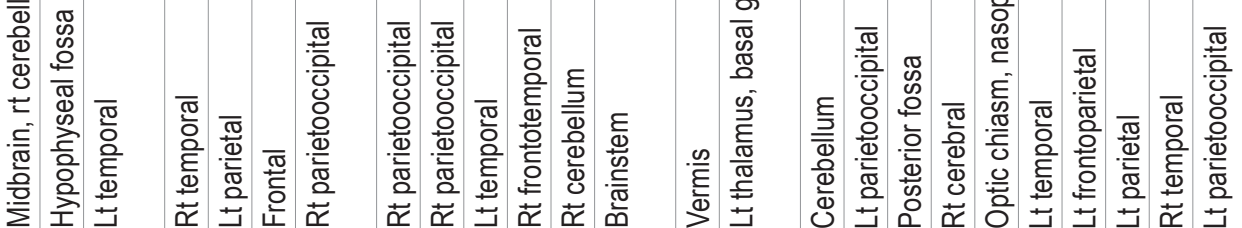

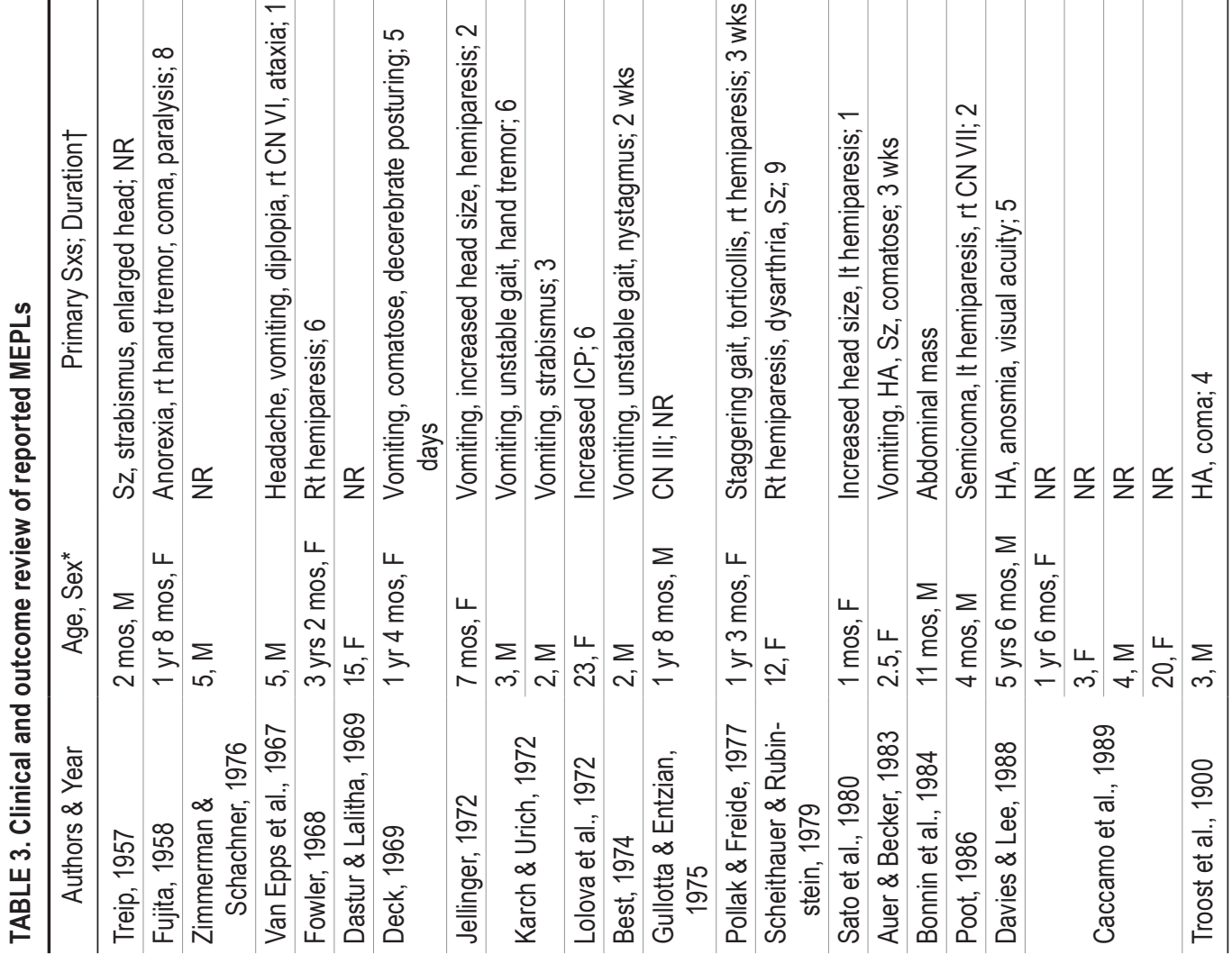




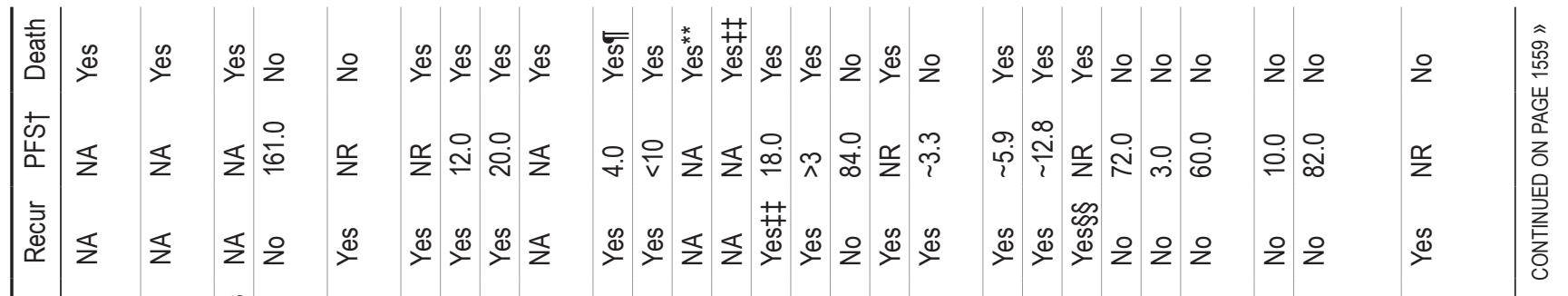

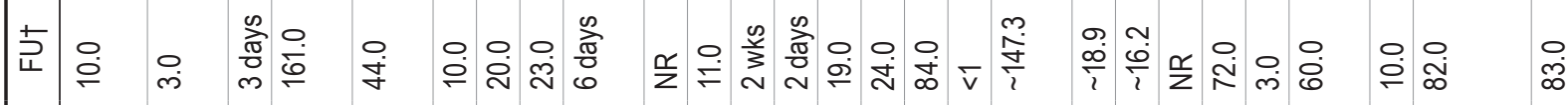

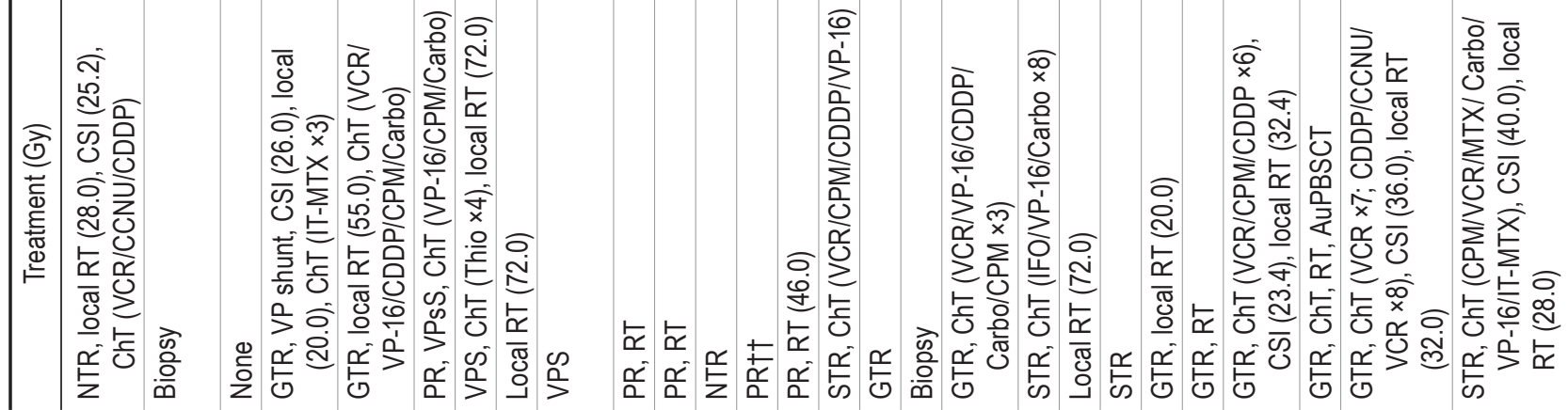
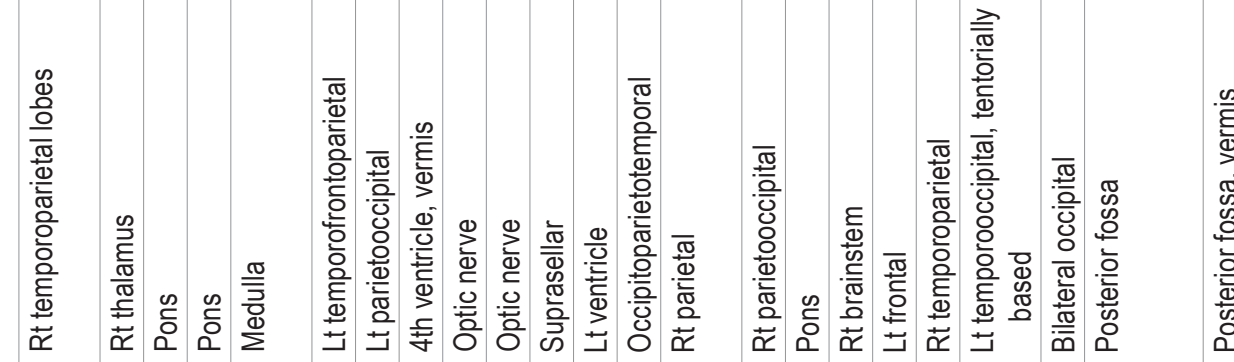

$$
\text { क्. }
$$

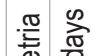

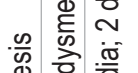

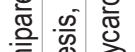

焉

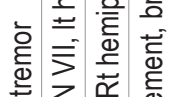

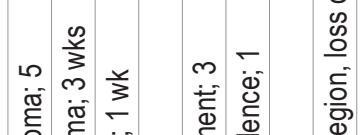

空

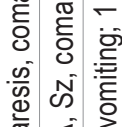

总

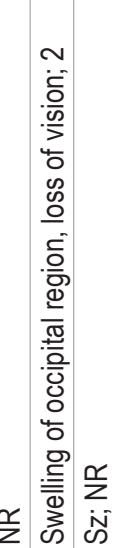

ㅁㄷ士무

용

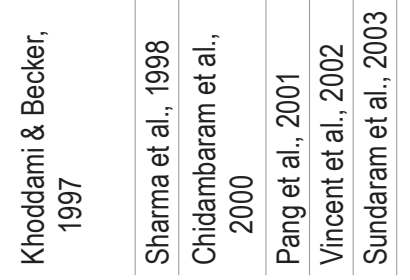

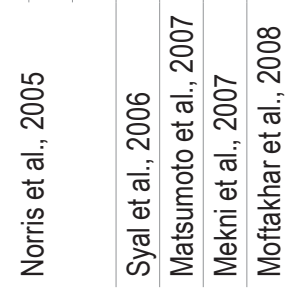

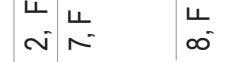


months), and the most common preoperative symptoms were motor weakness $(n=5)$ and vomiting $(n=5)$, followed by headache $(n=4)$, vertigo $(n=3)$, and ataxia, abnormal gait, and paresthesia in 2 patients each (Table 1). Two patients underwent ventriculoperitoneal shunt placement before admission to our institution. On admission, the preoperative KPS scores ranged from 40 to 80 , with a mean of $65 \pm 11$.

\section{Radiographic Evaluation and Preoperative Diagnosis}

A preoperative MRI scan was unavailable in 1 patient (case 11) because she required emergency surgery due to a progressively deteriorating condition (coma) caused by cerebral herniation and significant intracranial hypertension. A contrast-enhanced CT scan showed a right frontal lesion with a mass effect and ring-shaped nonhomogeneous enhancement. Overall, the lesion locations included the frontal lobe $(n=3)$, parietal lobe $(n=3)$, temporal lobe $(n$ $=2)$, occipital lobe $(n=2)$, brainstem $(n=3)$, cerebellum $(\mathrm{n}=1)$, and other locations (the fourth ventricle, left basal ganglia, and pineal region in 1 patient each). Multiple lobe involvement was observed in 3 cases. Eight lesions were supratentorial, and the other 4 lesions were infratentorial. With regard to the side of the lesion, 7 lesions were on the right side, 4 lesions were medially located, and only 1 lesion was on the left. The morphology was classified as completely solid $(n=2)$, solid with sporadic cysts $(n=$ $4)$, mixed solid-cystic $(n=1)$, and mixed cystic-solid ( $n=$ 5) according to the preponderance of solid or cystic components. Perilesional edema was significant or moderate in 7 lesions and 1 lesion, respectively, while the remaining 4 patients with sporadic cystic components $(n=2)$ or completely solid lesions $(n=2)$ had no observable edema. The lesion boundary was well demarcated in 6 patients and was poorly defined in the remaining 6 cases. Hydrocephalus was noted in 2 patients due to blockage of the mesencephalic aqueduct.

The signals of solid and cystic components are detailed in Table 1 and summarized in Supplemental Table 1. After administration of gadolinium, the solid component was nonhomogeneous, homogeneous, and had no enhancement in $9(81.8 \%)$ cases, $1(9.1 \%)$ case, and 1 case, respectively, and the capsule wall was enhanced in 5 cases $(45.5 \%)$. Intralesional hemorrhage occurred in 1 patient (Fig. 1). The mean equivalent lesion size was $4.2 \mathrm{~cm}$ (range $1.1-7.6 \mathrm{~cm}$ ), and the mean of the greatest dimension was $4.8 \mathrm{~cm}$. Preoperative diagnoses were established based on radiological data and were inaccurate in all cases, including 3 cases (25\%) of glioma, 2 cases $(16.7 \%)$ each of ependymomas and astrocytomas, and 1 case $(8.3 \%)$ each of glioblastoma, ganglioglioma, choroid plexus papilloma, pilocytic astrocytoma, and pineoblastoma.

\section{Surgical Findings}

The surgical approach was individualized in each case (Table 2). Lesions were abundantly, moderately, and mildly vascularized in 6,5, and 1 patients, respectively. During lesion dissection, the surgical plane was undefined in 9 lesions and clear in only 3 lesions. The consistency was soft in 3 cases and subject to suction, tenacious in 3 cases, and mixed soft-tenacious in 6 cases. The solid component was 


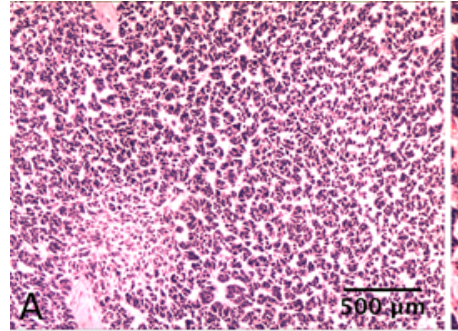

Hematoxylin-eosin $\times 100$

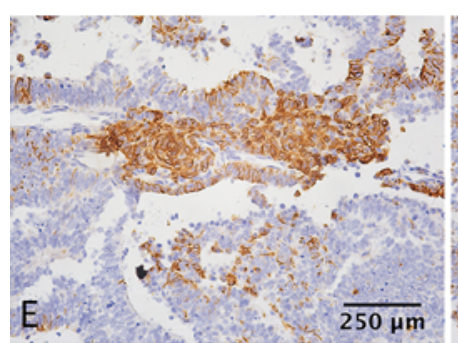

Cytokeratin $\times 200$

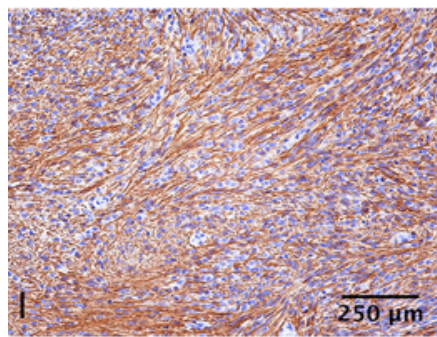

GFAP $\times 200$

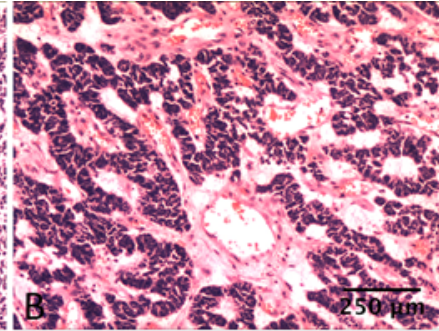

Hematoxylin-eosin $\times 200$

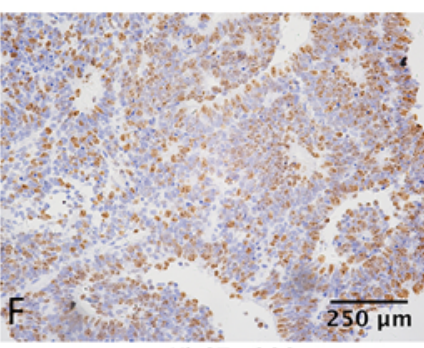

$\mathrm{Ki}-67 \times 200$

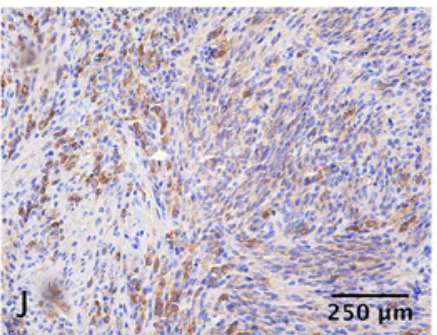

MAP- $2 \times 200$

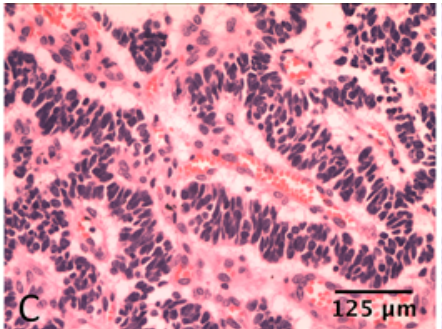

Hematoxylin-eosin $\times 400$

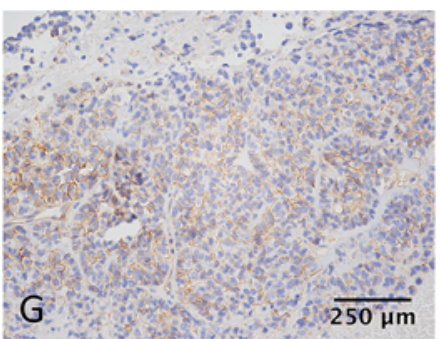

CD99 $\times 200$

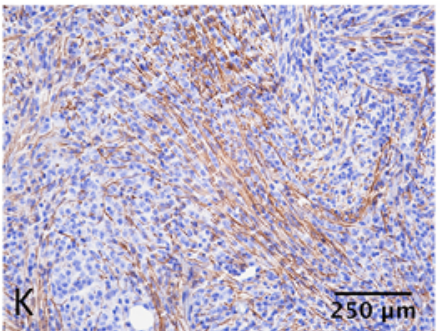

Nestin $\times 200$

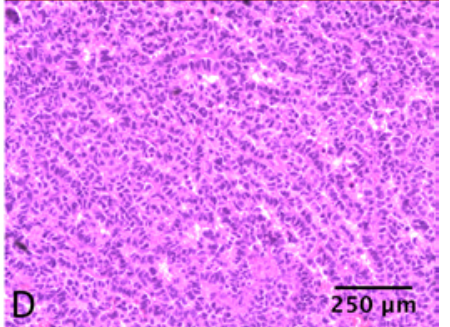

Hematoxylin-eosin $\times 200$

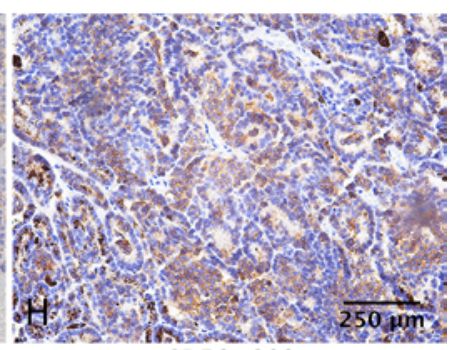

CD56 $\times 200$

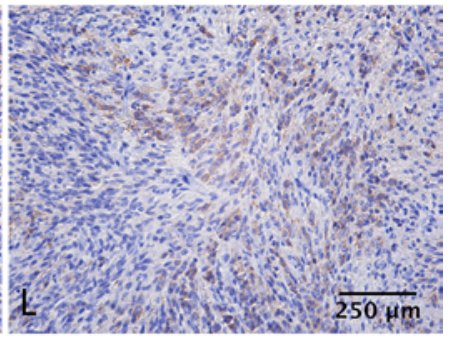

Synaptophysin $\times 200$

FIG. 2. Histopathological examination revealed the typical morphology of MEPL. A-D: Based on H \& E staining, MEPL displayed multipotential cellular lineages including a full spectrum of glioneuronal differentiation, and showed the structure of the primitive neuroepithelium arranged in tubular, ribbon-like, and/or papillary rosette formations lined by mitotic, pseudostratified columnar cells that resemble embryonic neural tube. E: Cytokeratin was focally presented in the neural tube-like structures. F: The Ki-67 proliferation index was calculated approximately up to $50 \%$. G and H: Diffuse membranous CD99 and CD56 expression in neoplastic cells. I: GFAP immunoreactivity was evident in abundant primitive cells close to neuroepithelial structures. J: Neoplastic cells were strongly diffusely positive for MAP-2. K: Strong nestin immunoreactivity was seen in the epithelial structures. L: Local synaptophysin immunoreactivity was expressed. Figure is available in color online only.

visually gray in 3 cases, gray-red in 6 cases, and mauve, light yellow, and gray-yellow in 1 case each. The fluid of the cystic component in 6 cases of either mixed cysticsolid $(\mathrm{n}=5)$ or solid-cystic $(\mathrm{n}=1)$ morphology was xanthochromic in various shades; however, similar findings were not identified in the other cases $(n=4)$ with cystic degeneration. Intraoperative blood loss ranged from 100 to $800 \mathrm{ml}$ with a mean volume of $320.8 \pm 224.1 \mathrm{ml}$. Based on postoperative MRI scans, GTR and STR were achieved in $10(83.3 \%)$ and $2(16.7 \%)$ patients, respectively, and surgical morbidity occurred in 3 patients.

\section{Surgical Outcomes}

At discharge, the mean KPS score was $72.5 \pm 13.6$ (range 50-90), which was improved, stabilized, or worsened in 7,3, and 2 patients, respectively, compared to the preoperative KPS score. Postoperative adjuvant radiotherapy was administered to 7 patients $(58.3 \%)$, among whom 4 patients (33.3\%) received chemotherapy; the treatment protocol is detailed in Table 1. The dose of the fractionated local radiation (cases 1, 7, and 9-12) ranged from 12.4
Gy to 58.2 Gy with mean and median doses of 38.2 Gy and $40.4 \mathrm{~Gy}$, and the CSI doses in 2 patients (cases 4 and 7) were 40.0 Gy and $30.6 \mathrm{~Gy}$, respectively. The mean total dose was $42.8 \mathrm{~Gy}$. The chemotherapy regimens were similar in cases 9 and 10, with 6 cycles of vincristine, cyclophosphamide, and cisplatin. In case 7, chemotherapy included 6 cycles of etoposide and carboplatin, 2 cycles of lomustine, and 6 cycles of cyclophosphamide and vincristine. In case 12 , the patient received 6 cycles of cyclophosphamide, vincristine, etoposide, and cisplatin, and 2 cycles of semustine. Given the agents used, the side effects were noted in these 4 cases, including bone marrow depression of varied degrees $(n=4)$, gastrointestinal toxicity (nausea, vomiting, and anorexia) $(\mathrm{n}=2)$, dermal toxicity (alopecia, erythra, and dermatitis) $(n=2)$, and hepatotoxicity $(n=1)$. All side effects were tolerable and eased after the chemotherapy course, except bone marrow depression required administration of recombinant human granulocyte colonystimulating factor.

After a median follow-up duration of 21.7 months (range 11.1-111.4 months), 6 patients experienced recur- 
TABLE 4. Summary of clinical characteristics of medulloepitheliomas from the literature and our series*

\begin{tabular}{|c|c|c|c|c|}
\hline \multirow[b]{2}{*}{ Variable } & \multicolumn{2}{|c|}{ Prior Studies $(n=55)$} & \multirow[b]{2}{*}{ Current Series } & \multirow[b]{2}{*}{ Overall } \\
\hline & No. of Available Cases & Value & & \\
\hline Mean age, yrs & 55 & $4.1 \pm 5.1$ & $15.7 \pm 11.9$ & $6.2 \pm 8.1$ \\
\hline $\operatorname{Sex}(M: F)$ & 45 & $22 / 23$ & $5 / 7$ & $27 / 30$ \\
\hline Chief complaint & 49 & & & \\
\hline Vomiting & & $20(40.8)$ & $5(41.7)$ & $25(41.0)$ \\
\hline $\mathrm{HA}$ & & $17(34.7)$ & $4(33.3)$ & $21(34.4)$ \\
\hline Motor weakness & & $12(24.5)$ & $5(41.7)$ & $17(27.9)$ \\
\hline Sz & & $10(20.4)$ & $1(8.3)$ & $11(18.0)$ \\
\hline Ataxia & & $8(16.3)$ & $2(16.7)$ & $10(16.4)$ \\
\hline Oculomotor deficit & & $5(10.2)$ & $1(8.3)$ & $6(9.8)$ \\
\hline Mean Sx duration, mos & 31 & $2.7 \pm 2.6$ & $2.1 \pm 3.3$ & $2.6 \pm 2.8$ \\
\hline Regions involved by lesions & 55 & & & \\
\hline Frontal/temporal/parietal/occipital & & $7 / 12 / 17 / 10$ & $3 / 2 / 3 / 2$ & $10 / 14 / 20 / 12$ \\
\hline Brainstem/cerebellum/other & & $7 / 8 / 12$ & $3 / 1 / 3$ & $10 / 9 / 15$ \\
\hline Multiple regions & & $18(32.7)$ & $3(25.0)$ & $21(31.3)$ \\
\hline Supra-/infratentorial & 55 & $38 / 17$ & $8 / 4$ & $46 / 21$ \\
\hline Side, It/rt/median & 48 & $19 / 15 / 14$ & $1 / 7 / 4$ & $20 / 22 / 18$ \\
\hline Mean lesion size, $\mathrm{cm} \dagger$ & 12 & $5.5 \pm 2.0$ & $4.8 \pm 2.0$ & $5.1 \pm 2.0$ \\
\hline Solid/solid-cystic & 21 & $6 / 15$ & $2 / 10$ & $8 / 25$ \\
\hline Median Ki-67/MIB-1 & 3 & $47.3 \%$ & $35.4 \%$ & $37.8 \%$ \\
\hline GTR/non-GTR & 51 & $13 / 38$ & $10 / 2$ & $23 / 40$ \\
\hline Pts w/ RT & 51 & $25(49.0)$ & $7(58.3)$ & $32(50.8)$ \\
\hline Pts w/ ChT & 51 & $15(29.4)$ & $4(33.3)$ & $19(30.2)$ \\
\hline No. of pts w/ relapse & 33 & $23(69.7)$ & $6(50.0)$ & $29(64.4)$ \\
\hline Mean PFS duration, mos & 16 & $8.8 \pm 7.0$ & $7.5 \pm 5.4$ & $8.5 \pm 6.5$ \\
\hline Mean FU, mos & 46 & $23.9 \pm 36.7$ & $31.5 \pm 28.8$ & $25.5 \pm 35.1$ \\
\hline Death & 48 & $34(70.8)$ & $6(50.0)$ & $40(66.7)$ \\
\hline
\end{tabular}

pts = patients.

Values are presented as the number of patients (\%) unless stated otherwise. Mean values are presented as the mean \pm SD.

* Unavailable data were excluded from the review.

† Greatest dimension.

rence, including 1 case of intracranial dissemination, with a median PFS time of 5.5 months (range 4.6-17.9 months). Two patients (cases 1 and 3 ) received treatment for recurrence but unfortunately died even after salvage treatment. Overall, these 6 patients with recurrence died of this disease and had a median survival time of 13.9 months (range 11.1-30.2 months). At the follow-up evaluation, the KPS scores of these surviving patients were 100 in 5 patients and 70 in 1 patient.

\section{Pooled Analysis of Individual Patient Data}

Between January 1957 and July 2017, 55 cases of MEPL were reported (Tables 3 and 4), including 22 males and 23 females (in the remaining 10 cases, sex was not reported) with a mean and median age of 4.1 years and 2.0 years (range 35 weeks' gestation to 35 years), respectively. ${ }^{1,3-39} \mathrm{In}$ 49 patients with detailed preoperative symptoms, vomiting ( $n=20,40.8 \%)$ was the most common symptom, followed by headache $(\mathrm{n}=17,34.7 \%)$, motor weakness $(\mathrm{n}=12$, $24.5 \%)$, seizure $(\mathrm{n}=10,20.4 \%)$, and ataxia $(\mathrm{n}=8,16.3 \%)$.
The duration of symptoms ranged from 0.1 to 9 months (median 2.0 months).

Lesions predominantly involved the parietal lobe $(\mathrm{n}=$ $17,30.9 \%)$, followed by the temporal lobe $(\mathrm{n}=12,21.8 \%)$, occipital lobe $(n=10,18.2 \%)$, cerebellum $(n=8,14.5 \%)$, frontal lobe $(n=7,12.7 \%)$, and brainstem $(n=7,12.7 \%)$. Multiple-region involvement was observed in 18 cases $(32.7 \%)$. The majority of cases were supratentorial $(n=38$, $69.1 \%)$, and the others were infratentorial $(\mathrm{n}=17,30.9 \%)$. GTR, near-total resection, STR, and PR were achieved in 13 (23.6\%), 2 (3.6\%), 5 (9.1\%), and 13 (23.6\%) cases, respectively. Five patients (9.1\%) underwent biopsy, and 11 patients $(20.0 \%)$ did not undergo resection. Treatment was not detailed in 4 patients $(7.3 \%)$, and the extent of resection was not reported in another 2 patients (3.6\%). Radiotherapy was administered to 25 patients $(49.0 \%)$, and the median dose was 53.2 Gy (range 20.0-72.0 Gy). Fifteen patients $(29.4 \%)$ received chemotherapy, and the regimens are detailed in Table 3. The treatment modalities used are summarized in Supplemental Table 1. 
Li et al.

TABLE 5. Cox regression model for risk factors predicting PFS

\begin{tabular}{|c|c|c|c|c|c|c|c|c|}
\hline \multirow[b]{2}{*}{ Variables } & \multirow{2}{*}{$\begin{array}{c}\text { No. of Pts } \\
\text { w/ PFS Data }\end{array}$} & \multirow{2}{*}{$\begin{array}{c}\text { No. w/ } \\
\text { Event (\%) }\end{array}$} & \multirow{2}{*}{$\begin{array}{l}1-Y r \\
\text { PFS }\end{array}$} & \multirow{2}{*}{$\begin{array}{l}5-Y r \\
\text { PFS }\end{array}$} & \multicolumn{2}{|c|}{ Univariate Analysis* } & \multicolumn{2}{|c|}{ Multivariate Analysis* } \\
\hline & & & & & $\mathrm{HR}(95 \% \mathrm{Cl})$ & $p$ Value & $\mathrm{HR}(95 \% \mathrm{Cl})$ & $\mathrm{p}$ Value \\
\hline Overall & 38 & $22(57.9)$ & 52.8 & 36.3 & & & & \\
\hline Age, per 1-yr increase & & & & & $0.963(0.908-1.020)$ & 0.196 & & \\
\hline Age $\geq 6$ yrs & 16 & $6(37.5)$ & 73.3 & 57.8 & Reference & & Reference & \\
\hline Age $<6$ yrs & 22 & $16(72.7)$ & 37.6 & 20.1 & $3.169(1.227-8.184)$ & 0.017 & $1.473(0.502-4.326)$ & 0.481 \\
\hline GTR & 21 & 7 (33.3) & 69.6 & 63.8 & Reference & & Reference & \\
\hline Non-GTR & 17 & $15(88.2)$ & 31.4 & 6.3 & $4.343(1.754-10.753)$ & 0.001 & $5.537(2.114-14.501)$ & $<0.001$ \\
\hline RT & 25 & $12(48.0)$ & 69.4 & 42.7 & Reference & & Reference & \\
\hline No RT & 13 & $10(76.9)$ & 23.1 & 23.1 & 2.505 (1.065-5.891) & 0.035 & 3.553 (1.389-9.091) & 0.008 \\
\hline RT dose, per 1-Gy increase & $31 \dagger$ & & & & $0.984(0.967-1.002)$ & 0.080 & & \\
\hline $\mathrm{ChT}$ & 14 & $5(35.7)$ & 60.6 & 60.6 & Reference & & & \\
\hline No ChT & 24 & $17(70.8)$ & 48.2 & 22.7 & $2.313(0.850-6.291)$ & $>0.99$ & & \\
\hline Treatment modality & & & & & 1.684 (1.266-2.239) & $<0.001$ & & \\
\hline GTR + RT + ChT & 8 & $1(12.5)$ & 87.5 & 87.5 & Reference & & & \\
\hline GTR + (RT or ChT) & 8 & $3(37.5)$ & 71.4 & 53.6 & $3.710(0.384-35.848)$ & 0.257 & & \\
\hline GTR alone & 5 & $3(60.0)$ & 40.0 & 40.0 & $2.394(0.771-7.440)$ & 0.131 & & \\
\hline Non-GTR + RT + ChT & 2 & $1(50.0)$ & 0 & 0 & $1.906(0.755-4.814)$ & 0.172 & & \\
\hline Non-GTR + (RT or ChT) & 11 & $10(90.9)$ & 45.5 & 9.1 & $1.865(1.112-3.128)$ & 0.018 & & \\
\hline Non-GTR alone or none & 4 & $4(100)$ & 0 & 0 & $1.713(1.098-2.673)$ & 0.018 & & \\
\hline
\end{tabular}

Boldface type indicates statistical significance.

${ }^{*}$ Forward stepwise (likelihood ratio).

$\dagger$ A total of 31 patients with detailed radiation dose for analysis.

TABLE 6. Cox regression model for risk factors predicting OS

\begin{tabular}{|c|c|c|c|c|c|c|c|c|}
\hline \multirow[b]{2}{*}{ Variable } & \multirow{2}{*}{$\begin{array}{l}\text { No. of Pts } \\
\text { w/ OS Data }\end{array}$} & \multirow{2}{*}{$\begin{array}{l}\text { Death } \\
(\%)\end{array}$} & \multirow{2}{*}{$\begin{array}{l}1-Y r \\
O S\end{array}$} & \multirow{2}{*}{$\begin{array}{l}5-Y r \\
\text { OS }\end{array}$} & \multicolumn{2}{|c|}{ Univariate Analysis* } & \multicolumn{2}{|c|}{ Multivariate Analysis* } \\
\hline & & & & & $\operatorname{HR}(95 \% \mathrm{Cl})$ & $p$ Value & $\mathrm{HR}(95 \% \mathrm{Cl})$ & $\mathrm{p}$ Value \\
\hline Overall & 58 & $38(65.5)$ & 53.9 & 29.2 & & & & \\
\hline Age, per 1-yr increase & & & & & $0.910(0.852-0.973)$ & 0.006 & & \\
\hline Age $\geq 6$ yrs & 18 & $6(33.3)$ & 94.4 & 60.3 & Reference & & Reference & \\
\hline Age $<6$ yrs & 40 & $32(80.0)$ & 35.6 & 15.1 & $4.768(1.962-11.586)$ & 0.001 & $2.591(0.887-7.567)$ & 0.082 \\
\hline GTR & 22 & $5(22.7)$ & 95.0 & 72.1 & Reference & & Reference & \\
\hline Non-GTR & 36 & $33(91.7)$ & 28.9 & 5.8 & $10.012(3.816-26.267)$ & $<0.001$ & $9.089(3.008-27.460)$ & $<0.001$ \\
\hline RT & 30 & $14(46.7)$ & 71.2 & 44.0 & Reference & & Reference & \\
\hline No RT & 28 & $24(85.7)$ & 35.7 & 14.3 & $3.033(1.562-5.892)$ & 0.001 & 3.126 (1.447-6.751) & 0.004 \\
\hline RT dose, per 1-Gy increase & $50 \dagger$ & & & & $0.980(0.966-0.993)$ & 0.004 & & \\
\hline ChT & 19 & $7(36.8)$ & 77.4 & 58.0 & Reference & & Reference & \\
\hline No ChT & 39 & $31(79.5)$ & 42.6 & 15.8 & $3.498(1.532-7.985)$ & 0.003 & $3.621(1.498-8.753)$ & 0.004 \\
\hline Treatment modality & & & & & $2.531(1.806-3.548)$ & $<0.001$ & & \\
\hline GTR + RT + ChT & 9 & $1(11.1)$ & 87.5 & 87.5 & Reference & & & \\
\hline GTR + (RT or ChT) & 8 & $1(12.5)$ & 100 & 80.0 & $1.069(0.067-17.118)$ & 0.962 & & \\
\hline GTR alone & 5 & $3(60.0)$ & 100 & 40.0 & $2.245(0.723-6.974)$ & 0.162 & & \\
\hline Non-GTR + RT + ChT & 5 & $3(60.0)$ & 50.0 & 25.0 & $2.087(0.979-4.451)$ & 0.057 & & \\
\hline Non-GTR + (RT or ChT) & 13 & $12(92.3)$ & 53.8 & 7.7 & $1.931(1.156-3.226)$ & 0.012 & & \\
\hline Non-GTR alone or none & 18 & $18(100)$ & 5.6 & 0 & $2.004(1.324-3.033)$ & 0.001 & & \\
\hline
\end{tabular}

${ }^{*}$ Forward stepwise (likelihood ratio).

† A total of 50 patients had radiation dose for analysis. 

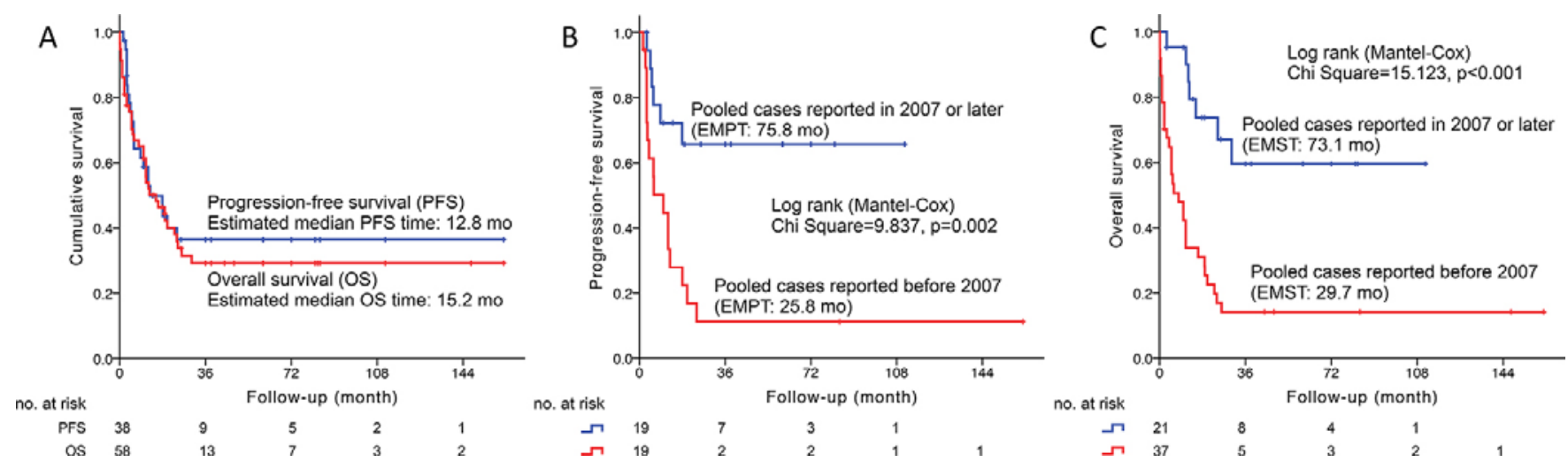

FIG. 3. Kaplan-Meier analyses illustrating the PFS $(n=38)$ and OS $(n=58)$ of MEPL based on the literature and our cohort $(\mathbf{A})$, and showing the significantly different PFS (B) and OS (C) in patients reported before 2007 or later. This confounding factor was taken into consideration when performing the multivariate analysis. Chi-square values were obtained by log-rank (Mantel-Cox) testing during the Kaplan-Meier curve analysis. EMPT = estimated median PFS time; EMST = estimated median survival time. Figure is available in color online only.

After a median follow-up duration of 10.0 months (range 2 days to 161.0 months), for cases that provided available information with regard to progression and death, the recurrence and mortality rates were $69.7 \%(23 / 33)$ and $70.8 \%$ (34/48), respectively.

\section{Statistical Analysis: Primary Results of PFS and OS}

The PFS and OS evaluation included our cohort and prior cases that had information available for analysis (Tables 5 and 6). The actuarial PFS rates at 1 and 5 years were $52.8 \%$ and $36.3 \%$, respectively, and the actuarial OS rates at 1 and 5 years were $53.9 \%$ and $29.2 \%$, respectively (Fig. 3). The estimated median survival times for PFS and OS were 12.8 and 15.2 months, respectively. A univariate Cox regression model identified age less than 6 years (HR 3.169, 95\% CI 1.227-8.184; p = 0.017) (Fig. 4A), non-GTR (HR 4.343, 95\% CI 1.754-10.753; p = 0.001) (Fig. 4B), and no radiotherapy treatment (HR 2.505, 95\% CI 1.065-5.891; $\mathrm{p}=0.035$ ) (Fig. 4C) as risk factors for poor PFS. Other factors, including sex (HR $0.839,95 \%$ CI $0.345-2.038 ; \mathrm{p}=0.698$ ), duration of symptoms (HR 1.071, 95\% CI 0.927-1.238; $\mathrm{p}=0.350$ ), and location (infratentorial vs supratentorial) (HR 1.054, 95\% CI 0.429-2.589; $\mathrm{p}=0.908)$ were not significant, and neither was chemotherapy (Fig. 4D). A multivariate Cox regression model verified that non-GTR (HR 5.537, 95\% CI 2.114-14.501; $\mathrm{p}<0.001$ ), and no radiotherapy treatment (HR 3.553,95\% CI 1.389-9.091; $\mathrm{p}=0.008)$ were independent adverse factors, but age was no longer a significant factor $(p=0.481)$ for PFS. Treatment modalities, as described in Table 5, were significantly associated with PFS (HR 1.684, 95\% CI 1.266-2.239, $\mathrm{p}<0.001)$. The different PFS among the various treatment modalities was significant at 5 years and sharply declined from $87.5 \%$ to $9.1 \%$.

Significant factors for OS identified by the univariate Cox regression model are detailed in Table 6 (Fig. 4). Both increased age by 1 year (HR $0.910,95 \%$ CI $0.852-0.973$; $\mathrm{p}=0.006)$ and increased radiation dose by 1 Gy (HR $0.980,95 \%$ CI $0.966-0.993 ; p=0.004$ ) favored OS, and the treatment modality type was also significantly associ- ated with OS, as the 5-year OS gradually decreased when the treatment modality changed from GTR+RT+ChT to non-GTR alone or none. Sex (HR 0.613 , 95\% CI $0.305-$ $1.232 ; \mathrm{p}=0.169$ ), duration of symptoms (HR $0.978,95 \%$ CI $0.864-1.107 ; \mathrm{p}=0.722$ ), and location (HR 1.501, 95\% CI $0.788-2.861 ; \mathrm{p}=0.217$ ) were not related to OS. A multivariate Cox regression model demonstrated that non-GTR (HR 9.089, 95\% CI 3.008-27.460; p < 0.001) (Fig. 4F), no radiotherapy (HR 3.126, 95\% CI 1.447-6.751; $\mathrm{p}=0.004$ ) (Fig. 4G), and no chemotherapy (HR 3.621, 95\% CI 1.498$8.753 ; \mathrm{p}=0.004)($ Fig. $4 \mathrm{H})$ were independent adverse factors for OS.

The effect of publication time of prior studies on the outcome was evaluated using ROC curve analysis, and 2007 was considered the cutoff that was adequately illustrated by the Kaplan-Meier survival curve (Fig. 3B and C). After adjustment for the confounding factor of publication time, non-GTR (HR 3.703, 95\% CI 1.234-11.110; p $=0.020)$ and no radiotherapy (HR 3.306, 95\% CI $1.285-$ 8.502; $\mathrm{p}=0.013$ ) remained risk factors for PFS. Non-GTR (HR 9.309, 95\% CI 3.003-28.862; $\mathrm{p}<0.001$ ), no radiotherapy (HR 3.750, 95\% CI 1.766-7.960; $\mathrm{p}=0.001$ ), and no chemotherapy (HR 3.193, 95\% CI 1.324-7.697; p = 0.010) remained risk factors for OS.

\section{Discussion}

MEPL is an unusual neoplastic entity that frequently affects the pediatric population. Due to the paucity of data regarding MEPL, the prognosis of MEPL has been generally described as dismal, with a propensity for progression, recurrence, and dissemination..$^{22}$ Based on our series and prior studies, we found that preoperative clinicoradiological data are less reliable and not sufficient for accurate diagnosis, and pathological examination should be performed to determine diagnosis. GTR and radiotherapy were independent favorable factors that significantly improved both PFS and OS, and chemotherapy only benefited OS. These results validated the modality of GTR plus radiochemotherapy, which is meaningful in clinical practice given these adjustable factors. 

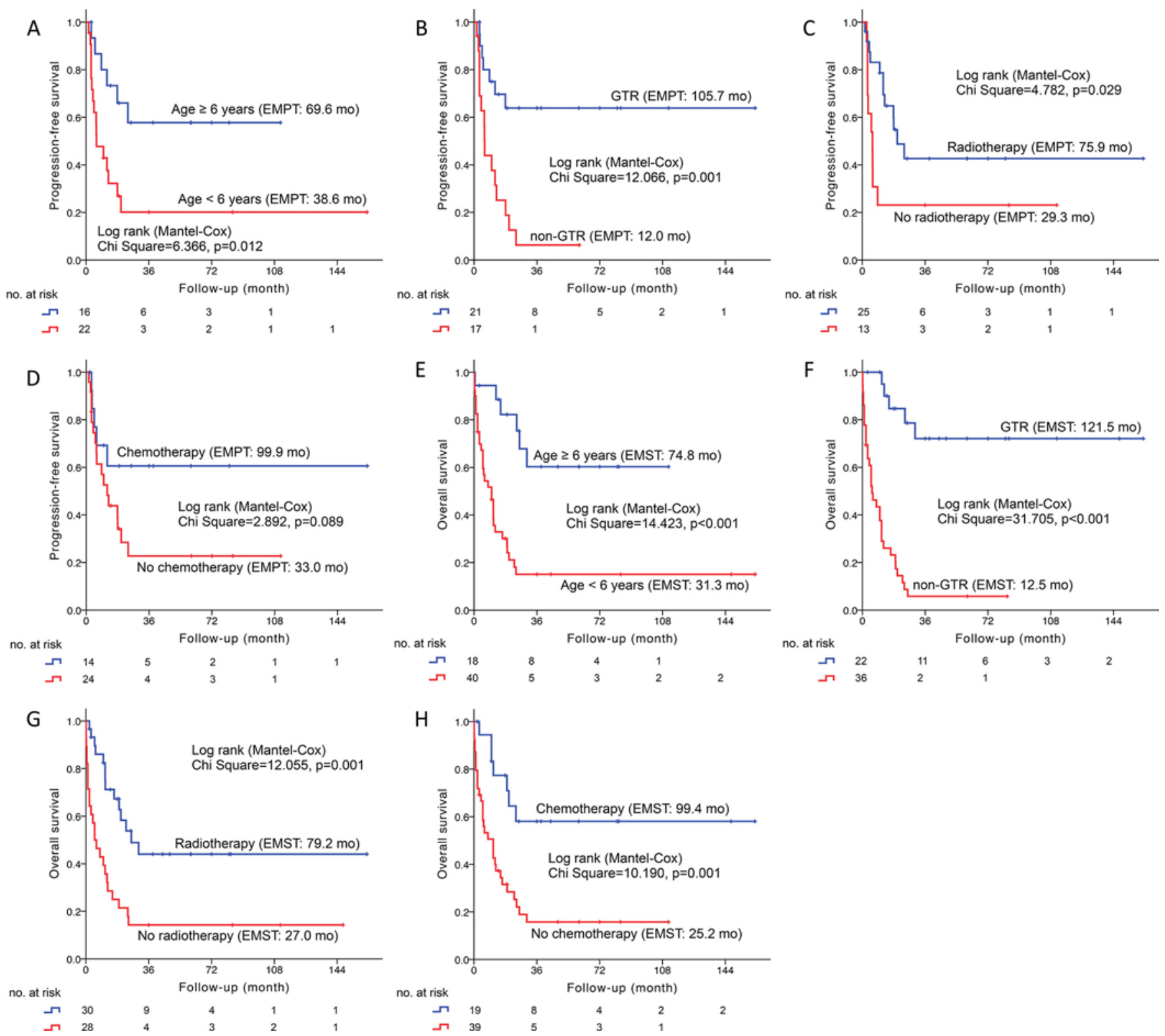

FIG. 4. Kaplan-Meier survival curves illustrating the different PFS (A-D) and OS $(E-H)$ in patients with risk factors. Patients younger than 6 years $(\mathbf{A})$, who did not undergo GTR $(\mathbf{B})$, or had no radiotherapy $(\mathbf{C})$ had a significantly worse PFS. Although the different PFS in patients with or without chemotherapy was not significant, the survival curve showed a poor outcome trend and shortened survival time in patients who did not undergo chemotherapy (D). The OS was significantly worse in patients younger than 6 years $(\mathbf{E})$, those who did not undergo GTR $(\mathbf{F})$, and had no radiotherapy $(\mathbf{G})$ or chemotherapy $(\mathbf{H})$. Chi-square values were obtained by log-rank (Mantel-Cox) testing during the Kaplan-Meier curve analysis. Figure is available in color online only.

\section{Clinicoradiological Features}

The median age of 3.0 years in this pooled cohort $(\mathrm{n}=$ 67 ) indicates the pediatric propensity of MEPL. However, patients older than 10 years have been reported. $6,9,19,21,29,31$ No sex predominance was observed. Occasionally, some uncommon symptoms, such as increased head size, ${ }^{16,30,35}$ anorexia, ${ }^{13}$ and torticollis, ${ }^{23,27}$ were reported. The similar mean symptom duration of 2.7 months in prior cases and 2.1 months in our series indicated the rapid deterioration of the disease. Unlike medulloblastomas with an infratentorial preference, MEPL was usually supratento- rial. ${ }^{22}$ Moftakhar et al. ${ }^{22}$ stated that the most common site involved, in order of frequency, was temporal, followed by parietal, occipital, and frontal lobes, which was slightly different from our findings (Table 4).

The radiological features have been rarely described in prior studies. The morphology was reported in only 21 cases (38.2\%), among which 6 cases were solid, , , 18,22 , 26,3411 cases were solid-cystic, $1,5,7,10,15,18,20,29,32,33$ and 4 cases were cystic-solid. ${ }^{24,28,37}$ Combined with our cases, it could be speculated that cystic degeneration is a common trait of MEPL; however, the proportion of lesions with cystic 
components versus solid components varied greatly. After administration of gadolinium in 14 prior cases, MEPL usually presented with nonhomogeneous enhancement (n $=8), 5,20,22,24,26,29,32,33$ but no enhancement $(\mathrm{n}=3)$ was seen in studies by Hayase et al., ${ }^{15}$ Mekni et al., ${ }^{21}$ and Syal et al., ${ }^{34}$ which is similar to case 5 from our series. The radiological characteristics of MEPL are nonspecific and cannot directly imply the diagnosis of MEPL or distinguish MEPL from other solid-cystic lesions.

\section{Progression-Free Survival and Risk Factors}

Due to the malignancy of MEPL, progression occurs inevitably without systemic treatment, and the rate is extraordinarily high. The median PFS duration in 16 patients with detailed data of progression was 6.0 months (range 1.5-24.0 months). ${ }^{8,17-19,23,25-27,31,36,37}$ In situ recurrence was the most common recurrence pattern, and extensive dissemination, ${ }^{34}$ extracranial metastasis, ${ }^{37}$ and intracranial metastasis $^{8}$ were also observed in a minority of cases, the latter pattern being similar to our case 1 . Among 10 patients with PFS, 5,10,15,20-24,29,38 9 patients received GTR, 8 patients received radiotherapy, and 6 patients received chemotherapy, which emphasizes the importance of GTR combined with adjuvant treatment, with GTR having the most striking impact. ${ }^{24}$ Radiation regimens were only detailed in 4 of these 10 cases, including CSI (doses of 23.4, 36.0, and 26.0 Gy) plus local radiation (doses of 32.4, 32.0, and $20.0 \mathrm{~Gy}$ ) in 3 cases, ${ }^{22-24}$ and local radiotherapy $(20.0$ Gy) in 1 case. ${ }^{20}$ In addition, CSI was used in another 5 patients ${ }^{1,12,17,23,24}$ (dose range 25.2-41.0 Gy), among whom only 1 survived. CSI was used in 2 of our patients (cases 4 and 7) with radiation doses of 40.0 and $30.6 \mathrm{~Gy}$, and fortunately, no progression or metastasis was observed. Autologous peripheral blood stem cell transplantation (AuPBSCT), as a part of initial treatment planning, was used only in 2 cases with relatively short follow-up durations. ${ }^{5,15}$ However, its side effects have been thoroughly discussed by Moftakhar et al., ${ }^{22}$ and further investigation is needed to confirm whether AuPBSCT is superior to conventional therapy or if it can be used as an alternative to radiotherapy in pediatric patients..$^{15,22}$

Chemotherapy regimens, which were diverse, included intrathecal methotrexate injection, ${ }^{23,24} 4$ cycles of thiotepa, ${ }^{23}$ and/or traditional regimens comprising vincristine, ${ }^{7,15,22-26}$ etoposide, ${ }^{15,23-26}$ cyclophosphamide ${ }^{7,15,22-26 /}$ ifosfamide, ${ }^{25}$ lomustine, ${ }^{23,24}$ and/or carboplatin ${ }^{23-25} /$ cispla- $^{2}$ tin. ${ }^{7,15,22-26}$ Overall, the combination of vincristine/cisplatin/cyclophosphamide was the most frequently used.,15,22, 23,25,26 In cases with a favorable PFS, Moftakhar et al. ${ }^{22}$ used 6 cycles of vincristine/cyclophosphamide/cisplatin in a 9-year-old girl that was parallel to the treatment in 2 of our patients (cases 9 and 10), Hayase et al. ${ }^{15}$ administered 5 cycles of vincristine/etoposide/cisplatin/cyclophosphamide to a 2-year-old boy, and Müller et al. ${ }^{24}$ administered 7 cycles of vincristine and 8 cycles of lomustine/cisplatin/ vincristine in a 7-year-old girl. Because the detailed treatment regimens varied among studies, no consensus could be established; nevertheless, GTR plus radiochemotherapy was preferred, and CSI plus local radiation was advocated, for which long-term PFS durations of 60.0, 82.0, and 161.0 months have been observed..$^{22-24}$
Risk factors for PFS have not been evaluated before. A multivariate Cox regression (Table 5) confirmed non-GTR and no radiotherapy as independent risk factors for PFS. The association between the increased radiation dose and PFS ( $p=0.080)$ shows the potential remarkable effect of a high radiation dose in tumor control, which was demonstrated in 2 progression-free cases reported by Müller et al. ${ }^{24}$ and Moftakhar et al. ${ }^{22}$ after total doses of $68.0 \mathrm{~Gy}$ and $55.8 \mathrm{~Gy}$, respectively. Otherwise, it was noted that the small sample size compromised the statistical confidence. An age less than 6 years significantly predicted poor PFS using the univariate model $(\mathrm{p}=0.017)$ but was no longer significant in the multivariate model because bivariate correlation analysis found higher proportions of GTR and radiotherapy in patients $\geq 6$ years.

\section{Overall Survival}

The OS of MEPL was discouraging and significantly associated with tumor progression. From the pooled data (Tables 1 and 3), the median and mean OS durations in 38 patients with specific data of survival duration were 7.0 and 9.5 months, respectively, and the actual mortality was $66.7 \%$ (40/60 cases), including 3 surgical deaths. ${ }^{8,11,32}$ The actuarial 3- and 5-year OS was reported to be $30 \% .{ }^{24}$ In 6 untreated cases reported by Treip, ${ }^{35}$ Fujita, ${ }^{13}$ Jellinger, ${ }^{16}$ Gullotta and Entzian, ${ }^{14}$ Sato et al., ${ }^{30}$ and Molloy et al., ${ }^{23}$ the median survival duration was only 2.0 months. In contrast, the median survival duration in patients receiving GTR plus radiotherapy or chemotherapy increased to 42.0 months ${ }^{15,19-21,25,29}$ and further increased to 60.0 months if they received GTR plus radiochemotherapy. ${ }^{522-24}$ Otherwise, in 10 patients surviving at least 36 months, ${ }^{15,19,20,}$ 22-25,38 9 benefited from GTR plus adjuvant treatment, and in our series, 5 of 6 survivors underwent GTR. These data emphasize the value of initial systemic treatment rather than reserving adjuvant treatment for recurrence or rendering residual/recurrence to secondary surgery, as only 4 (17.4\%) of 23 patients with recurrences survived, ,, $23-25$ and all of our patients with recurrences $(n=6)$ died.

It is worth reviewing the salvage treatment with which 4 patients with recurrences attained long-term survival. ${ }^{19,23-25}$ In a 17-month-old patient reported by Molloy et al., ${ }^{23}$ greater than 44 months survival was obtained after the patient received GTR, AuPBSCT, and cyclophosphamide, etoposide, and thiotepa cytoreduction after recurrence. In the series by Norris et al., ${ }^{25}$ a 28-month-old girl survived more than 147.3 months by undergoing GTR, AuPBSCT, etoposide, thiotepa, and carboplatin, and local radiation with a total dose of 59.4 Gy after regrowth. The third case of an 8-year-old girl reported by Müller et al. ${ }^{24}$ underwent radiotherapy (CSI and local radiation, total 68 Gy), vincristine, GTR, and 4 cycles of maintenance chemotherapy (cisplatin, lomustine, and vincristine) after recurrence, and complete remission was recorded after 83 months. The fourth case reported early in 1972 by Lolova et al. ${ }^{19}$ was a 23 -year-old woman who experienced 4 local recurrences and/or extracranial metastasis, each followed by GTR plus radiotherapy within 48 months of followup. The chemotherapy regimens were slightly different between these first 3 patients based on tailored treatment principles; however, it was evident that GTR was the com- 
mon point and had significant effects on OS. The effect of AuPBSCT on OS was considered promising by Hayase et al. ${ }^{15}$ but it remains poorly defined because only $2(40 \%)$ of 5 patients with recurrences with AuPBSCT survived. ${ }^{23,25}$

The independent risk factors for OS are controversial. Moftakhar et al. ${ }^{22}$ found that certain characteristics were predictive of more favorable outcomes, including a supratentorial location, no dissemination, GTR, radiotherapy, and chemotherapy, but cited that age and sex were not predictive of survival. Müller et al. ${ }^{24}$ reported 2 unadjusted risk factors (age less than 4 years and no GTR) for OS. The 5-year OS in patients with age greater than or equal to 4 years or GTR was $50 \%$ and $91 \%$, respectively. By contrast, it was $18 \%$ and $13 \%$ in patients with age less than 4 years or no GTR, respectively. However, they did not reveal other independent risk factors. With this evidence, our results (Table 6) indicated that chemoradiotherapy as an adjustable parameter should be tailored to all patients with a sufficient dose, if tolerable. We did not find that infratentorial location was a risk factor, which is similar to a prior study. ${ }^{24}$

\section{Proposed Treatment Paradigm}

The significantly different 5-year PFS of MEPL among these 6 treatment modalities (Table 5) supported an optimal protocol consisting of GTR plus radiochemotherapy. This protocol has been advocated by several studies ${ }^{20,22,24,29}$ and is similar to the protocol for high-risk medulloblastoma. ${ }^{24}$ Additionally, the significantly different OS stratified by treatment (Table 6) justifies the necessity of this protocol. Matsumoto et al. ${ }^{20}$ recommended excision of any surrounding brain tissue infiltrated by tumor cells. The extent of resection was dependent on the intraoperative findings, and if GTR was impossible due to location or early dissemination, the outcome was relatively poor regardless of chemoradiotherapy. Routine diagnostic workup, including CSF cytology and radiographs for clinical staging (presence of residual, metastasis, or not), is mandatory. Radiotherapy should include CSI and local radiation to reduce the chances of dissemination or intra- or extracranial metastasis.

Due to the rarity of the disease, there was no available clinical practice guideline published by National Comprehensive Cancer Network; therefore, in the pooled data the heterogeneity in treatment algorithms inevitably existed and should be underlined. As a result, some previously reported patients were treated by reference to the protocols for medulloblastoma and/or supratentorial primitive neuroectodermal tumors, including CSI with boosting to the primary brain site, similar chemotherapy regimens, and autologous stem cell reinfusion. Nevertheless, there are profound implications in terms of our results including: 1) We identified a general treatment protocol and recommended that was useful for both patients and clinicians. 2) Salvage treatments from pooled data were noted and evaluated that potentially benefited patients experiencing recurrence. Otherwise, the present study urges future research to propose further detailed, reliable radiotherapy and chemotherapy regimens pertinent to MEPL, and to design a staging system and risk rating system tailored to MEPL to achieve both homogeneous and individualized treatment.

\section{Limitations of the Present Study}

We cited treatment regimens of patients with favorable outcomes that might have significance for future cases, but poor outcome was often encountered even with aggressive treatment. Treatment varied among studies over a length of time, and we found a significantly different PFS and OS between patients treated before and after 2007. Publication time, as a confounding factor, was adjusted via a multivariate model, and the identified risk factors remained significant. Due to the rarity of MEPL, chemotherapy regimens could not be unified and should be individualized. Although there was no consensus regarding the treatment for recurrent MEPL, GTR plus chemoradiotherapy is recommended, and AuPBSCT may be attempted.

\section{Conclusions}

MEPL is a rare primitive neuroectodermal tumor with pediatric predominance, frequently radiological cystic degeneration, and poor clinical outcomes. GTR and radiotherapy were independent favorable factors for both PFS and OS. Chemotherapy significantly improved OS. Based on these adjustable factors, GTR plus chemoradiotherapy of a sufficient dose, if tolerable, is advocated as an optimal treatment for MEPL. Future studies with larger cohorts are necessary to verify our findings and to improve the extrapolation of these results.

\section{References}

1. Auer RN, Becker LE: Cerebral medulloepithelioma with bone, cartilage, and striated muscle. Light microscopic and immunohistochemical study. J Neuropathol Exp Neurol 42:256-267, 1983

2. Bailey P, Cushing H: A Classification of Tumors of the Glioma Group on a Histogenetic Basis with a Correlated Study of Progress. Philadelphia: JB Lippincott, 1926

3. Best PV: Posterior fossa medulloepithelioma. Report of a case. J Neurol Sci 22:511-518, 1974

4. Bonnin JM, Rubinstein LJ, Palmer NF, Beckwith JB: The association of embryonal tumors originating in the kidney and in the brain. A report of seven cases. Cancer 54:2137-2146, 1984

5. Buccoliero AM, Castiglione F, Rossi Degl'Innocenti D, Franchi A, Paglierani M, Sanzo M, et al: Embryonal tumor with abundant neuropil and true rosettes: morphological, immunohistochemical, ultrastructural and molecular study of a case showing features of medulloepithelioma and areas of mesenchymal and epithelial differentiation. Neuropathology 30:84-91, 2010

6. Caccamo DV, Herman MM, Rubinstein LJ: An immunohistochemical study of the primitive and maturing elements of human cerebral medulloepitheliomas. Acta Neuropathol 79:248-254, 1989

7. Chakrabarti I, Majumdar K, Giri A: Infratentorial medulloepithelioma with divergent differentiation: Possibly a predictor of poor outcome. J Pediatr Neurosci 7:142-145, 2012

8. Chidambaram B, Santosh V, Balasubramaniam V: Medulloepithelioma of the optic nerve with intradural extension-report of two cases and a review of the literature. Childs Nerv Syst 16:329-333, 2000

9. Dastur DK, Lalitha VS: Pathological analysis of intracranial space-occupying lesions in 1000 cases including children. 2 . Incidence, types and unusual cases of glioma. J Neurol Sci 8:143-170, 1969

10. Davies RP, Lee CS: Medulloepithelioma: MRI appearances. Australas Radiol 32:503-505, 1988 
11. Deck JH: Cerebral medulloepithelioma with maturation into ependymal cells and ganglion cells. J Neuropathol Exp Neurol 28:442-454, 1969

12. Fowler M: Embryonic ependymoma arising in a cerebral hemisphere. Cancer 21:1150-1156, 1968

13. Fujita S: Medulloepithelioma: its place in histological classification of neuroectodermal tumours. Acta Pathol Jpn 8:789-794, 1958

14. Gullotta F, Entzian W: [Clinical and morphological considerations concerning "medulloepithelioma" (author's transl).] Neurochirurgia (Stuttg) 18:193-199, 1975 (Ger)

15. Hayase T, Morimoto A, Kawahara Y, Yagi M, Kanai N, Nobusawa $\mathrm{S}$, et al: An infant with medulloepithelioma successfully treated by high-dose chemotherapy followed by autologous peripheral blood stem cell transplantation without radiotherapy. J Pediatr Hematol Oncol 37:e394-e398, 2015

16. Jellinger K: Cerebral medulloepithelioma. Acta Neuropathol 22:95-101, 1972

17. Karch SB, Urich H: Medulloepithelioma: definition of an entity. J Neuropathol Exp Neurol 31:27-53, 1972

18. Khoddami M, Becker LE: Immunohistochemistry of medulloepithelioma and neural tube. Pediatr Pathol Lab Med 17:913-925, 1997

19. Lolova I, Bojinov S, Ivanova A, Christov V: Cerebral medulloepithelioma. Histological and histochemical study of a case. J Neurol Sci 15:173-182, 1972

20. Matsumoto M, Horiuchi K, Sato T, Oinuma M, Sakuma J, Suzuki K, et al: Cerebral medulloepithelioma with long survival. Neurol Med Chir (Tokyo) 47:428-433, 2007

21. Mekni A, Ferchichi L, Kallel J, Braham E, Bellil K, Bellil $\mathrm{S}$, et al: Intracranial medulloepithelioma. A case report and review of the literature. Pathologica 99:11-14, 2007

22. Moftakhar P, Fan X, Hurvitz CH, Black KL, Danielpour M: Long-term survival in a child with a central nervous system medulloepithelioma. J Neurosurg Pediatr 2:339-345, 2008

23. Molloy PT, Yachnis AT, Rorke LB, Dattilo JJ, Needle MN, Millar WS, et al: Central nervous system medulloepithelioma: a series of eight cases including two arising in the pons. J Neurosurg 84:430-436, 1996

24. Müller K, Zwiener I, Welker H, Maass E, Bongartz R, Berthold F, et al: Curative treatment for central nervous system medulloepithelioma despite residual disease after resection. Report of two cases treated according to the GPHO Protocol HIT 2000 and review of the literature. Strahlenther Onkol 187:757-762, 2011

25. Norris LS, Snodgrass S, Miller DC, Wisoff J, Garvin J, Rorke LB, et al: Recurrent central nervous system medulloepithelioma: response and outcome following marrow-ablative chemotherapy with stem cell rescue. J Pediatr Hematol Oncol 27:264-266, 2005

26. Pang LM, Roebuck DJ, Ng HK, Chan YL: Sellar and suprasellar medulloepithelioma. Pediatr Radiol 31:594-596, 2001

27. Pollak A, Friede RL: Fine structure of medulloepithelioma. J Neuropathol Exp Neurol 36:712-725, 1977

28. Poot RD: Medulloepithelioma: first CT images. Neuroradiology 28:286, 1986

29. Ramesh AS, Anita M, Jitender S, Somanna S: Unusual occurrence of supratentorial medulloepithelioma in a young female. J Neurosci Rural Pract 5:261-264, 2014

30. Sato T, Shimoda A, Takahashi T, Daita G, Goto S, Takamura $\mathrm{H}$, et al: Congenital cerebellar neuroepithelial tumor with multiple divergent differentiations. Acta Neuropathol 50:143-146, 1980

31. Scheithauer BW, Rubinstein LJ: Cerebral medulloepithelioma. Report of a case with multiple divergent neuroepithelial differentiation. Childs Brain 5:62-71, 1979

32. Sharma MC, Mahapatra AK, Gaikwad S, Jain AK, Sarkar C: Pigmented medulloepithelioma: report of a case and review of the literature. Childs Nerv Syst 14:74-78, 1998

33. Sundaram C, Vydehi BV, Jaganmohan Reddy J, Reddy AK: Medulloepithelioma: a case report. Neurol India 51:546547,2003

34. Syal R, Reddy S J, Kumar R, Tyagi I, Abrar AA, Krishnani $\mathrm{N}$, et al: Unusual clinical and MRI features of a cerebellopontine angle medulloepithelioma. Case report and review of literature. Pediatr Neurosurg 42:299-303, 2006

35. Treip CS: Congenital medulloepithelioma of the midbrain. J Pathol Bacteriol 74:357-363, 1957

36. Troost D, Jansen GH, Dingemans KP: Cerebral medulloepithelioma-electron microscopy and immunohistochemistry. Acta Neuropathol 80:103-107, 1990

37. Van Epps RR, Samuelson DR, McCormick WF: Cerebral medulloepithelioma. Case report. J Neurosurg 27:568-573, 1967

38. Vincent S, Dhellemmes P, Maurage CA, Soto-Ares G, Hassoun J, Ruchoux MM: Intracerebral medulloepithelioma with a long survival. Clin Neuropathol 21:197-205, 2002

39. Zimmermann A, Schachner M: Nervous system antigen-5, an antigenic cell surface component of neuroectodermal origin. Brain Res 115:297-310, 1976

\section{Disclosures}

The authors report no conflict of interest concerning the materials or methods used in this study or the findings specified in this paper.

\section{Author Contributions}

Conception and design: all authors. Acquisition of data: Jia, D Li, Hao, L Wang, GL Li, JM Wang, Wu, LW Zhang. Analysis and interpretation of data: Jia, D Li, Hao, GL Li, JM Wang, Wu, LW Zhang, JT Zhang. Drafting the article: Jia, D Li. Critically revising the article: all authors. Reviewed submitted version of manuscript: all authors. Approved the final version of the manuscript on behalf of all authors: Jia. Statistical analysis: Jia, D Li, Hao. Administrative/technical/material support: all authors. Study supervision: Jia, D Li, Hao, GL Li, JM Wang, Wu, LW Zhang, JT Zhang.

\section{Supplemental Information \\ Online-Only Content}

Supplemental material is available with the online version of the article.

Supplemental Table 1. https://thejns.org/doi/suppl/10.3171/ 2018.1.JNS172509.

\section{Correspondence}

Wang Jia: Beijing Tiantan Hospital, Capital Medical University, Beijing, People's Republic of China. jiawangttyy@163.com. 